\title{
Über einen neuen Typus von sarkoiden Geschwülsten der Haut.
}

\author{
Von \\ C. Raseh, \\ Direktor der dermatolog. Poliklinik des \\ Kgl. Frederiks-Hospital in Kopenhagen. \\ F. Gregersen, \\ und ehem. 1. Assistent des pathol. Institutes \\ der Universität Kopenhagen.
}

(Hiezu Taf. XII.)

Die Patientin, welche die Veranlassung der folgenden Mitteilung gewesen ist, sah ich (C. R.) zum ersten Mal am 2. Oktober 1901 und stellte sie selbigen Tages der Dänischen dermatologischen Gesellschaft vor.

Die Krankengeschichte ist folgende:

Pat., eine 33jährige Dame, ist von gesunder Familie; der Vater ist an einer Herzkrankheit gestorben, ihre Mutter und Geschwister sind gesund. Selbst war sie früher stets gesund, eine ausgesprochene Nervosität und eine bewegliche Niere ausgenommen. Sie hat nie an andauerndem Husten oder Blatspeien gelitten, oder irgend andere Zeichen der Tuberkulose dargeboten. Vor 8 Jahren entstand auf der Dorsalseite des Zeigefingers an der linken Hand ein weißliches Geschwülstchen in der Haut, welches sie selbst, nachdem es ein Jahr bestanden, durch Ätzen mit Salpetersäure destruierte. Man findet eine runde weiße Narbe darnach, von ungefähr 1 Zentimeter im Diameter. Während 6 Jahren danach bemerkte sie nichts, im Laufe des letzten Jahres sind aber einige ähnliche Geschwülste an den Fingern der r. Hand aufgetreten. Diese Geschwülstchen begannen wie eine ganz kleine weißliche Hervorragung in der Haut; einige wachsen langsam bis auf einen Querschnitt von zirka 1 Zentimeter, andere verschwinden spontan, nachdem sie einige Monate bestanden haben, ohne deutliche Zeichen oder Narben zu hinterlassen. Keine Empiindlichkeit, kein Jucken oder sonstige subjektive Symptome.

Arch. f. Dermat. u. Syph. Bd. LXIV. 
Pat. ist blond, blaß und ziemlich mager, die Untersuchung bietet nichts Abnormes dar, die Affektion an den Fingern ausgenommen. Diese besteht aus 4 GeschwüIstchen, welche sich alle auf der Dorsalseite der Finger der rechten Hand befinden, An dem Zeigefinger, der 1. Phalanx und dem Gelenk, zwischen 1. and 2. Phalanx entsprechend befinden sich 2 Geschwülste, die erste $4 \mathrm{~mm}$, die zweite $1 \mathrm{~cm}$ im Diameter; an dem Mittelfinger findet man ein Geschwülstchen, dem Gelenk zwischen 1. und 2. Phalanx entsprechend, $5 \mathrm{~mm}$ an Diameter; an der Basis des 5 . Fingers eine Geschwulst, welche $1 \mathrm{~cm}$ an Diameter beträgt. In der Mitte zeigt sie eine fast völlige Regression, wodurch sie Ringform erhält. Die vom Ring umgebene Hautpartie ist glatt, schwach atrophisch. Alle Geschwülstchen sind von derber Konsistenz, die Farbe ist weißlich oder weißgelb, wie altes Elfenbein, mit glatter wachsartig glänzender Oberfäche. Sie haben ihren Sitz in der Substanz der Lederhaut und bewegen sich frei auf dem subkutane Bindegewebe. Als Rest einer der verschwundenen Geschwülstchen findet man am Mittelfinger einen linsengroßen Fleck, wo die Haut schwach atrophisch mit weniger ausgesprochener Furchenbildung ist als an den umgebenden Partien.

Pat, reiste gleich danach ins Ausland und ich habe sie später nicht wiedergesehen.

Am 30. Juli 1902 schreibt sie mir, daß alle Geschwülste, die sich später etwas vergrößert hatten, plötzlich im Verlaufe von einigen Tagen nach einem heftigen Fieberanfalle unbestimmter Natur Mitte Juli vollständig verschwanden mit Hinterlassung schwach rötlicher Flecken.

Mikroskopische Untersuchung. (F. G.)

Für die mikroskopische Untersuchung wurde ein Stück des sich an der Basis des 5. Fingers befindlichen ringförmigen Geschwülstchens exzidiert, in Formol-Müllerscher Flüssigkeit fixiert, in Alkohol gehärtet und in Paraffin eingebettet. Das Präparat wurde in 160 Serienschnitte zerlegt, teils nach van Giesons Methode (Hansens Modifkation), teils mit Hämatoxylin und Eosin gefürbt. Außerdem wurde Thioninfärbung, Färbung des elastischen Gewebes nach der UnnaTänzerschen Methode, Protoplasmafärbung nach Unna und Färbung nach Ziehl-Neelsens Methode angewandt.

In dem peripheren Teil der Geschwulst findet man in den ersten Schnitten in der Cutis propria verästelte, bluterfüllte Gefäße. Ihr Endothel ist natürlich und die Wandung ganz dünn. Sie sind von einer Zellverbrämung umgeben, teils aus Rundzellen bestehend, teils aus Zellen von dem Typus der Bindegewebszellen. Einige dieser Zellen baben längliche, spindelförmige schmale Kerne und ibr Aussehen weicht von den gewöhnlichen Bindegewebszellen nicht ab, andere sind plumper, mit rundlichen oder ovalen Kernen. Diese gefäBhaltigen Bindegewebsstränge liegen in der eigentlichen Cutis, während der Papillarkörper nichts Abnormes darbietet. Er ist durch eine normal aussehende dünne Bindegewebsschicht von den beschriebenen Zellsträngen geschieden. 
Folgt man diesen Strängen auf die eigentliche Geschwulstmasse zu, so findet man, daß die perivaskuläre Zellverbrämung an Breite zunimmt und eine dicke Scheide um die feinen Gefäße bildet. Vielerseits findet man eine nicht geringe Menge Rundzellen zwischen den Bindegewebszellen, deren Anzahl auch bedeutend vergrößert ist. Zwischen den Bindegewebsbündeln sind außerdem Streifen von Zellen befindlich, welche ganz dasselbe Gepräge haben wie die perivaskulär gelagerten. Diese Zelleninfiltration in den Lücken des Bindegewebes ist stärker ausgesprochen, je weiter man in der Schnittserie auf den zentralen Teil der Geschwulst kommt. Diese Zellaüge stehen in Verbindung mit den gefäßführenden Zellsträngen in der Peripherie der Geschwulst und enthalten mehrfach ganz feine Gefäße, welche nur aus einem einfachen Endothelrohr bestehen. Die Endothelzellen sind stets normal, ohne Zeichen von Proliferation.

Die Zellformen in dem zentralen Teil der Geschwulst siud ron außerordentlich mannigfacher Gestalt. Zellen mit randen oder ovalen Kernen findet man in überwiegender Menge, doch trifft man aufierdem Zellen mit birnförmigen, plump stabförmigen, gekrümmten oder sonstig geformten Kernen. Alle diese Kerne haben ein feingranuliertes Kernplasma, in welchem man einzelne oder wenige punktförmige distinkte Kernkörperchen autrifft. Der Zellkörper ist auch von sehr verschiedener Gestalt. Öfters ist er recht groß, mit vereinzelten protoplasmatischen Ausläufern versehen, die bald ziemlich kurz, bald lang und fein sind; oft ist der Zellkörper spindelförmig oder er bildet eine schmale Verbrämung um den Kern, schwach faserig am Rande.

Kernteilungsfiguren sind nicht vorhanden, aber viele Zellen enthalten 2 Kerne, deren einer oft in Verlängerung des anderen gestellt ist. Bisweilen trifft man größere Zellen, die bis 4 Kerne enthalten und teilweise einander decken; wirkliche Riesenzellen sind nirgends vorhanden.

Durch Anhäufen der beschriebenen Zellen werden die Bindegewebsbündel auseinander gesprengt und man findet sie als dünnere oder dickere Balken zwischen den Zellenanhäufungen, die sich ohne scharfe Grenzen in anscheinend normales Gewebe verlieren. In den Präparaten, die nach van Gieson-Hansen's Methode gefärbt sind, sieht man zwischen den Zellen ganz feine Bindegewebsfibrillen, eine spärliche Interzellularsubstanz zwischen ihnen bildend. Bei der Untersuchung mit der Immersionslinse scheint es, als ob diese feinen Bindegewebsfädchen sich aus Zellenaus läufern bilden würden.

Normale Bindegewebszellen trifft man nicht in den Zellenanhäufungen und es scheint überhaupt, als ob nicht allein die perivaskulären (Lymphraum-) Bindegewebszellea proliferieren, sondern daß auch die Bindegewebszellen in einiger Entfernung von den Gefäßen an dem Prozesse teilnehmen. Außer den bisher erwähnten Veränderungen sind in der Tiefe des Geschwulstgewebes unregelmäßig geformte Partien nachzuweisen, in welchen die Zellen zu Grunde gegangen sind. Die Nekrose wird stärker und tiefer, je weiter man in das Geschwulstgewebe dringt, sie tritt übrigens 
mit großer Unregelmäßigkeit auf, indem sie größere und kleinere Foci aufweist. An diesen Stellen findet man myxomatöse Metamorphose verbreitet, indem fadenartige Massen in der Umgebung der nekrotischen Partien vorbanden sind, welche mit Thionin Muzinreaktion ergehen. Die schleimige Degeneration der Zellen wird stärker, je weiter man in die zentrale Partie der Nekrosen kommt und die Muzinbildung im Zellenkörper wird augenscheinlich von einer Vernichtung der Zellen begleitet, indem die Konturen von diesen undeutlicher werden und verschwinden und die Kerne blasser werden oder sich in Körner auflösen und auseinanderfallen, so daß man im Zentrum der nekrotischen Partien nur fadenartige Muzinmassen findet, die hie und da Kernreste enthalten. Zwischen diesen Massen befinden sich Bindegewebsbündel, die sich scharf wie hellblane Balken zwischen den rotvioletten Schleimmassen abheben und demnach nicht myxomatös verändert zu sein scheinen. Der Übergang von den nekrotischen Partien zu dem lebenden Gewebe ist nicht scharf.

In den letzten Serienschnicten findet man die erwähnte Zellenproliferation nur an der einen Seite der Schnitte, während an der anderen Seite der Schnitte (welche der zentralen Regression in der ringförmigen Affektion entspricht) teils festes fibröses, teils loses fibrilläres Bindegewebe vorhanden ist. Diese letzte Partie scheint ungefähr wie normales Hautbindegewebe vaskularisiert und die Gefäße sind anscheinend ganz normal, wenn man einige Rundzellenanhäufungen in der Umgebung ausnimmt. In der Nähe der Zellenproliferationszone ist dieses Bindegewebe von stahförmigen Kernen und von länglichen schmalen Bindegewebszellen, die in Zügen verlaufen, durchsetzt. Hier sind die Bindegewebsbündel dicht gelagert mit schmalen länglichen Spalten; andererseit \& findet man das Bindegewebe loser and nicht so zellreich.

Der Übergang von der Zellenproliferationszone zu der beschriebenen Partie, welcbe nur um die Hälfte so dick wie der geschwulstinfiltrierte Teil der Haut ist, geschieht ganz gleichmäßig und gradweise.

Die Menge des elastischen Gewebes erscheint normal an den Stellen, wo keine Zellenproliferation vorhanden ist. Das subepitheliale Netz im Papillarkörper ist überall wohlerhalten und erscheint in orceingefärbten Schnitten als ein außerordentlich feinfadiger Plexus. An den Stellen, wo die Zellenproliferation nachzuweisen ist, fehlt das elastische Gewebe gänzlich, wo die Gewebsformation rein zellular ist. Es scheint auch in den nekrotischen Partien zu fehlen, obgleich die kollagenen Bündel hier nicht zu Grunde gegangen sind. Wo die Zellzüge vorhanden sind und wo das krankhafte Gewebe zwischen den Bindegewebsbündeln hevordrängt, findet man eine nicht unbedeutende Menge elastischen Gewebes, das an mehreren Stellen wie in ganz kleine Stücke zerbrochen erscheint. Andererseits und in den breiteren Bindegewebszügen sieht man es als kürzere und längere geringelte und gekrümmte, teils verästelte Fädchen.

Die kleinen Gefäße, um welche der periphere Teil der Zellenproliferation lokalisiert ist, enthalten kein elastisches Gewebe. An den größeren Gefäßen sind die Verhältnisse normal. 
Über einen neuen Typus v, sarkoiden Geschwälsten d. Haut. 341

An der Oberhaut wie an den Schweißdrüsen sind keine pathologischen Veränderungen vorhanden.

Nirgends beobachtet man Plasmazellen; die Ehrlich'schen Mastzellen findet man gelegentlich, hauptsächlich im Papillarkörper. Um die Gefäße der Schweißdrüsen trifft man einzelne. Zwischen den proliferirenden Zellen, in den Zellzügen und in den Zellanhäufungen findet man sie nicht.

Das subkutane Bindegewebe ist nur in ganz minimaler Menge vorhanden und nur an einigen Stellen, wo die Exzision in die tiefsten Schichten der Cutis reicht, an der Grenze des subkutanen Gewebes. Die Zellenproliferation scheint stets auf die Cutis selber beschränkt zu sein. Bakterien, speziell Tuberkelbazillen sind nicht vorhanden.

Das Resultat der histologischen Untersuchung weist also auf, daß man es mit einem Neoplasma zu tun habe, welches den Geschwülsten der Bindegewebsgruppe zuzurechnen ist und welches deutlich durch Proliferation der Zellen in den perivaskulären (peritelialen) Lymphräumen hervorzugehen scheint, obgleich man nicht mit Sicherheit ausschließen kann, daß auch Bindegewebszellen außerhalb der Gefäße an der Neubildung teilnehmen.

Daß die Geschwulst eine maligne Neubildung, ein Sarkom sein sollte, ist wenig wahrscheinlich; der klinische Verlauf spricht entschieden dagegen. Auch nicht die Struktur der Geschwulst weist mit Sicherheit auf Sarkom, wie es aus der Beschreibung hervorgeht; ferner muß man hervorheben, daß die Zellformen des Gewebes ihrer Polymorphie zum Trotz nicht in dem Grade von dem Typus der gewöhnlichen Bindegewebszellen abweichen, daß man die Geschwulst nach dem histologischen Bilde als ein perivaskuläres Sarkom hätte bezeichnen können. Es ist eine Geschwulst, die sich streng auf die Cutis selber beschränkt und hauptsächlich auf die Pars reticularis derselben. Bildet sie auch hier kein scharf abgegrenztes Knötchen, sondern zeigt eine Neigung das Gewebe in der Umgebung zu infiltrieren, so bricht sie doch nirgends durch den Papillarkörper hervor; das Geschwulstgewebe infiltriert ebenfalls das subkutane Gewebe nicht.

Man hat früher bei der histologischen Diagnose der Hautgeschwülste ein besonderes Gewicht auf das Verhalten des elastischen Gewebes gelegt, namentlich bei der Diagnose Sarkom.

Unna schreibt z. B. (Histopathologie): „Das elastische Gewebe geht jedoch gleich anfangs schon in der Übergangs- 
zone zugrunde; nur an den Gefäßen finden sich hier und da im ausgebildeten Sarkomgewebe noch Reste derselben."

In unserem Falle fehlt das elastische Gewebe nur da, wo die Zellproliferation am dichtesten ist, in großen Partien des Geschwulstgewebes findet man es aber fast unverändert. Nach den Untersuchungen von Polack, Daniels jst es indessen bewiesen, daß3 das elastische Gewebe nicht nur in Sarkomen rorhanden ist, sondern da $\beta$ es sich hier sogar neu bilden kann. Dasselbe Verbalten gilt für die Entwicklung des elastischen Gewebes im Narbengewebe (Jores). Das Verhalten des elastischen Gewebes kann also keine sichere Auskunft über die Natur der Neubildung geben; die Berechtigung, sie aus der Gruppe der Sarkome auszuschließen, muß man daher in den oben erwähnten Momenten suchen.

Vergleichen wir den Verlauf der Krankheit, das makroskopische Aussehen und das histologische Bild, so kommt es uns wahrscheinlich vor, daß wir es mit einer wohl abgegrenzten Krankheitsform von besonderer Art $\mathrm{zu}$ tun haben. Beim Studium der Literatur findet man auch, daß ganz ähnliche Krankheitsfälle früher beschrieben wurden.

Der erste von diesen Fällen ist von Colcott Fox mitgeteilt (Dermatol. soc. of London, 13. Feber 1895, Br. j. of d. 1895 p. 91) unter dem Titel "ringed eruption on the fingers". Pt. war ein 1ljähriges Mädchen, an deren Beugeseite des linken Ringfingers eine ovale ringförmige Affektion beobachtet wurde mit glatter, runder, hervorragender Kante, von weißer Farbe und teigartiger Konsistenz, $1 / 8$ Zoll in der Breite und $1 / 16$ Zoll in der Höhe. Die Haut in der Mitte hatte normales Ausseben oder war vielleicht etwas röter wie normal. Der Rand war etwas empfindlich gegen Druck. Keine sonstigen subjektiven Symptome. An dem kleinen Finger der rechten Hand fand man einen ähnlichen Ring nur ein wenig kleiner. Der Rand bestand hier aus getrennten Knötchen. Die Affektion befand sich in der Cutis selber. Die Krankheit hatte 2 wei Monate vorher als ein kleines Knötchen angefangen und war allmälig gewachsen. Dem Kinde fehlte sonst nichts, F. konnte keine bestimmte Diagnose stellen, glaubte aber, da $\beta$ er es mit einer indolenten Form von Entzündung zu tun habe, von Rheumatismus abhängung und mit Radcliffe Crockers Erythema elevatum dintinum rerwandt. Es fehlt uns in diesem Falie an histologischen Aufklärungen und an Mitteilung über den weiteren Verlauf.

Der nächste Fall ist von W. Dubreuilh mitgeteilt. (Sur un cas d'éruption circinée chronique de la main, Soc. derm. et syph. 19. Avril 1895, Ann. derm. et syph. 1895 p. 355.) Patientin war eine 33jährige Dame, 
mager und nervös, sonst aber gesund. Ausgesprochene rbeumatische Disposition. In ihrer Kindheit bis zum 16. Jahre hatte Patientin viel an Erythema Pernio gelitten. Die Affektion an den Fingern hatte fünf Jahre gedauert und hatte als kleine, derbe weiße Knötchen an der Radialseite beider Zeigefinger und an der Cubitalseite des linken Daumens begonnen. Indem sie in der Mitte schwanden, wachsen sie langsam, so daß sie Ringe mit exzentrischem Wachstum bildeten. In den letzten 4-5 Monaten wurden einige neue Eftloreszenzen unter der Form von linsengroßen Knoten an beiden Zeigefingern und Mittelfingern beobachtet.

Status praesens: Die Hände kühl, leicht cyanotisch; an beiden sind zahlreiche Narben nach Frostbeulen vorhanden. An der Dorsalseite des ersten Fingergliedes an Zeigefinger and Mittelfinger beider Hände findet man die frischen Knötchen, die sich als linsengroße, blasse, runde Hervorragungen ergeben, von glatter und leicht schuppender Oberhaut bedeckt. Sie sind von recht derber Konsistenz und erinnern an ein Keloid; sind in die Cutis eingelagert, sind indolent und gegen die Unterlage verschieblich. Das eine der Knötchen hat die Form eines Halbkreises. An dem linken Zeigefinger befindet sich eine der älteren Affektionen, die aus einem großen länglichen Ring bestehend, die normale Haut umgibt. Der Rand ist derb, blaß, von Keloidkonsistenz, an beiden Seiten von einer schmalen, schwach violettfarbenen Verbrämung abgegrenzt. Ferner findet man vier andere Ringe und Halbringe an anderen Fingern. Nach Behandlung von einigen Monaten mit Vida ls Mennig- und Zinnoberpflaster trat bedeutende Regression in den meisten Effloreszenzen ein.

Die histologische Untersuchung ergab: normale Oberhaut, in der Cutis eine diffuse Infiltration von Zellen, die wie fixe Bindegewebszellen aussahen. Man fand sehr wenig Mastzellen und keine Riesenzellen. Das Zelleninfiltrat beschränkte sich ausschließlich auf die Pars reticularis, indem sowohl der Papillarkörper als das sabkutane Bindegewebe völlig normal waren. Keine deutliche Veränderungen des Bindegewebes oder des elastischen Gewebes wurde beobachtet. Die Zellen waren zwischen den Bindegewebsbalken infiltrirt. In der zentralen Partie findet man eine kleine Zone, wo die Kerne sich nicht fürben lassen (Nekrose). Die Bindegewebsfibrillen und die elastischen Fasern scheinen in dieser Partie ohne Unterbrechung zu verlaufen.

Der dritte Fall ist von. I a mes Galloway beschrieben (Lichen annularis, a ringed eruption on the extremities, Br. j. d. 1899 p. 221).

Patient war ein 10jähriger, blasser, etwas kränklicher Junge, dessen Hautaffektion drei Jahre bestanden hatte. Sie fing als kleine Knötchen an den Fingerknöcheln an, die später zu Ringen mit blassen, elfenbeinartigen erhöhten Rändern heranwachsen. Der Kand ist 1-2 $\mathrm{mm}$ hoch und zirka $3 \mathrm{~mm}$ breit, glatt, ohne scheinbare Veränderungen in der Oberhaut. Der Untersuchung mit bloßem Auge stellt sich die vom Ringe umschlossene Hautpartie normal dar, mit der Lupe aber sieht man, daß die normalen Furchen teils fehlen und dab schwache Zeichen von Atrophie vorhanden sind. An beiden Händen fand man zerstreut neun teils sehr 
große Ringe oder Halbringe. Bei der histologischen Untersuchung waren in der Pars reticularis dichte Zellenanhäufungen nachzuweisen, von denen kleinere Infiltrationen längs der kleinen Gefäße ausstrablten. An einigen Stellen verläuft die Zelleninfiltration in die Papillarschicht und an einigen Stellen findet man sie, wenn anch weniger dicht in dem subkutanen Gewebe. Die meisten Zellen sind von langer und spindelförmiger Gestalt und den fixen Bindegewebszellen ähnlich. Man findet nur sehr wenig Rundzellen und sparsame Mastzellen. In dem zentralen Teil des Infiltrates ist Nekrose vorhanden. Die Bindegewebsfibrillen und die elastischen Fasern sind in ihrer Anordnung gestört, sind aber überall im Infiltrate nachzuweisen. In der Oberhaut wurden leichte sekundäre Veränderungen beobachtet.

Galloway identifiziert seinen Fall miţ den zweí oben erwähnten von Colcott Fox und Dubreuilh. Einige Fälle, von Hutchinson und R. Crocker publiziert, von chronischen roten Plaques an den Extremitäten werden auch von $G$ allow a y zitiert als wahrscheinlicherweise mit seinen $F$ ällen verwandt; diese weisen aber unserer Meinung nach entweder so große Differenzen von den angeführten Fällen auf, oder sind so unvollständig beschrieben, daß sie hier am besten außer Betracht gelassen werden. G. faßt das Leiden als einen chronisch inflammatorischen Prozeß auf, möglicherweise von Arthritis und Rheumatismaus abhängig.

Die Fälle von Dubreuilh und Galloway sind in allen Hauptpunkten dem unsrigen so ähnlich, daß wir keinen Zweifel hegen, daß es sich bei allen drei Patienten um dieselbe Affektion handelt. Diese ist durch eine sehr langsame Entwicklung ron weißen oder blaßgelben Infiltraten charakterisiert, die geneigt sind Ringe zu bilden und die sich ausschließlich auf die Finger beschränken. Das Leiden hat in allen drei Fällen hauptsächlich seinen Sitz in der Pars reticularis und das Infiltrat besteht fast ausschließlich aus jungen Bindegewebszellen mit Neigung zu zentraler Nekrose in den Knötchen. In keinem der Fälle sind Momente nachzuweisen, die mit Sicherheit Auskunft über die eigentliche Natur des Leidens erteilen.

Wie eingangs erwähnt, spricht die ganze klinische Entwickelung gegen die Diagnose Sarkom und diese Diagnose wird überhaupt weder von Dubreuilh noch von Galloway diskutiert. Dagegen weist der mikroskopische Befund, besonders in unserem Falle, darauf hin, daß die Affektion mit den noch wenig bekannten, sogenannten benignen $\mathrm{s}$ a $\mathrm{r}$ ko i d en Geschwülsten der Haut verwandt ist, wie sie 
Über einen neuen Ty pus v. sarkoiden Geschwülsten d. Haut. 345

von B o eck beschrieben wurden (Norsk Magasin f. Lægev. 1899 Nr. 11 und Archiv für Dermatologie und Syphilis, Festschrift Kaposi 1900.) Es besteht hinsichtlich des Ausgangspunktes des Prozesses und zu einem gewissen Grade in dem Charakter der Zellen eine Übereinstimmung zwischen dem unsrigen Fall und dem ron Boeck beschriebenen. Man kann doch keineswegs in unserem Falle die Zellen als "epithelioid“ bezeichnen, da dieser Typus durchaus nicht besonders vorherrschend ist, wenn auch einzelne "epithelioide" Zellen zwischen den anderen beschriebenen Formen befindlich sind. Wie in unserem Falle ist auch in dem Falle ron Boeck eine Degeneration der Zellen vorhanden, man findet aber nicht angegeben, welcher Art diese gewesen ist; ferner ist das Gefäßendothel normal und die Geschwulst hat denselben Ausgangspunkt wie in unserem Falle.

Auf der anderen Seite bestehen indessen so viele sowohl histologische als klinische Differenzen zwischen unserem und dem Fall von Boeck, daß man die beiden Fälle nicht identifizieren kann. Man kann nur sagen, daß die Affektionen wahrscheinlich verwandter Natur sind.

Bis auf weiteres sind wir deshalb darauf angewiesen, unseren Fall (mit dem von Dubreuilh und Galloway gemeinschaftlich) als einen neuen Typus in die Gruppe der benignen sarkoiden Geschwülste der Haut zu stellen.

Die histologische Untersuchung ist im pathologischen Institute der Universität ausgeführt worden, dessen Chef, Herrn Prof. J. Fibiger, wir unseren verbindlichsten Dank sagen. 


\section{Erklärung der Abbildungen auf Taf. XII.}

Fig. 1. Schnitt durch den zentralen Teil der Geschwulst: faserige Schleimmassen (a), schwach angedeutete Bindegewebsbalken (c), in der Peripherie starke Zellenanhäufungen zum Teile feine Gefäße enthaltend (b). - Thioninfärbung. Zeiß Okul, 2, Obj. H. H. Vergrößerung 50.

Fig. 2. Eine perivaskuläre Zellinfiltration an der Peripherie der Geschwulst. Das Endothel ist normal. Das Gefäß von Bindegewebszellen und Rundzellen umgeben. - Hämatoxylin-Eosin, Zeiß Ok. 2, Obj. D. D. Vergrößerung 240.

Fig. 3. Blutkapillare in einer größeren Zellenanbäufung, von Bindegewebszellen und einzelnen Leukocyten (a) umgeben. - Polychromes Methylenblau, Protoplasmafärbung. Zeiß, homog. Immersion 1/12 Ok. 2. Vergrößerung $6 \$ 0$. 


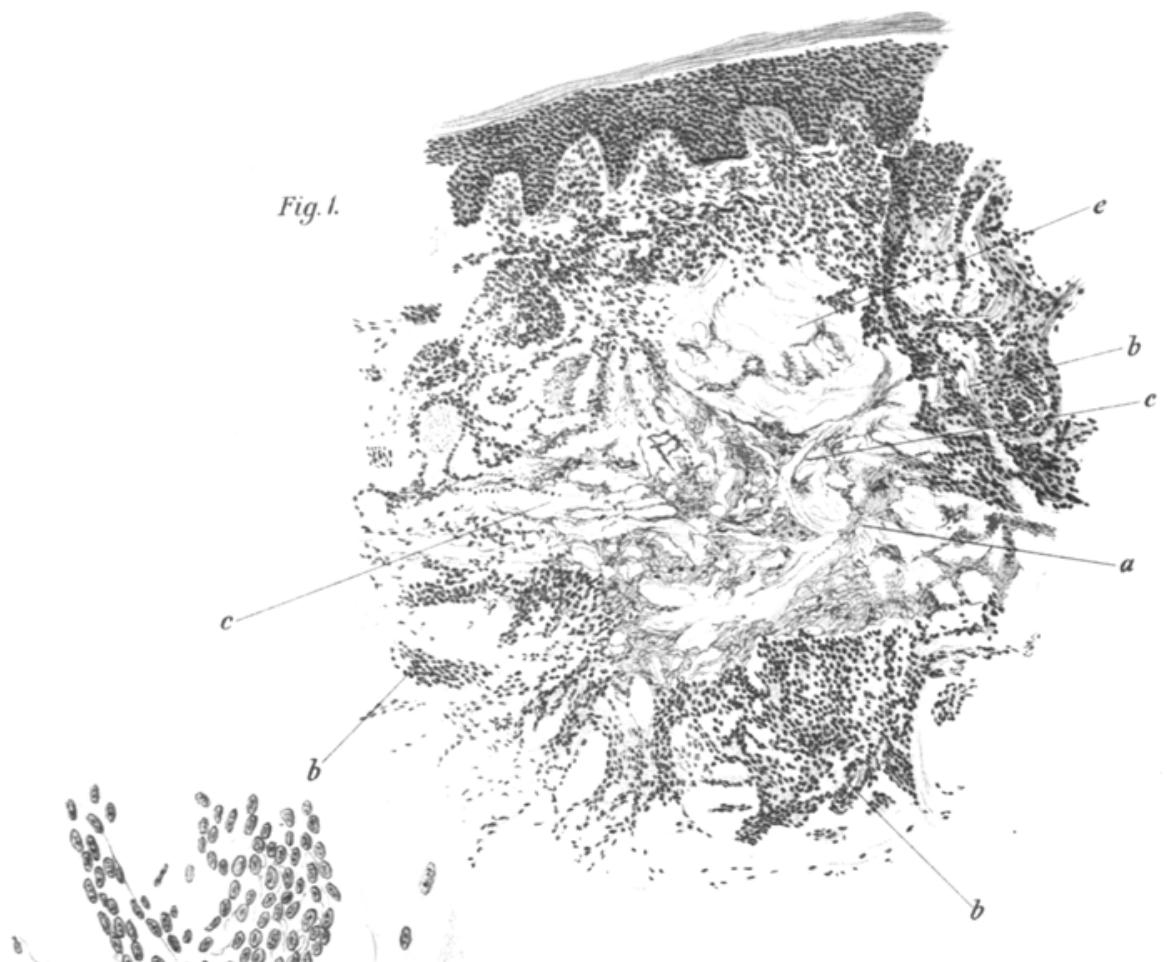

$\because-1,10 \% \%$

1 i. 18

Linol iojogos

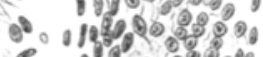

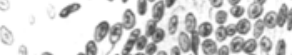

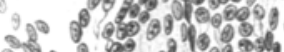

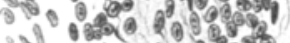

is 1090

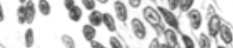

$\rightarrow \theta_{0}$ irgieso

Fig. 2.

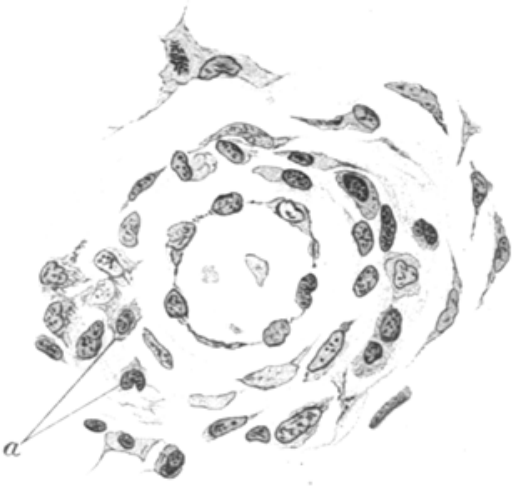

Fig. 3 .
- $10 \%$

$$
\text { 1 } 1 \text { \% }
$$

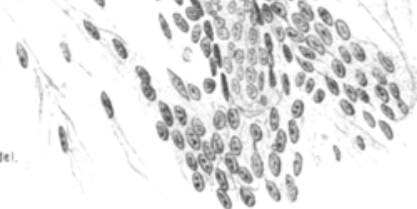

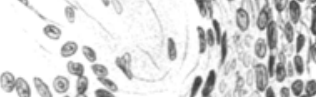

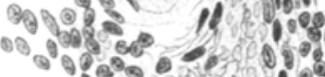

$\sin ^{\circ} 0$

$V$

C. Rasch u.F. Gregersen: ̈̈ber einen neuen Typus von sarkoiden Geschwülsten der Haut. 